\title{
DAS PRAETERITUM IN DEN SÜDDEUTSCHEN MUNDARTEN.
}

Unter die sprachlichen erscheinungen, welche die mundarten des südlichen und nördlichen Deutschlands trennen, ist vor allem der verlust der einfachen praeteritalformen im mittelund oberdeutschen $\mathrm{zu}$ rechnen. An die stelle dieser formen sind die des periphrastischen perfects getreten; fur hatte, spielle, nahm u. s. w. sagen also die slidlichen mundarten hal gehabt, hat gespielt, hat genommen. Man suchte in verschiedener weise diesen verlust zu erklären. In meiner dissertation Beiträge zur syntax der Mainzer mundart habe ich folgende erklärung gegeben. Das mhd. unbetonte $e$ ist in oberd. und md. mundarten lautgesetzlich weggefallen, und daher sind viele formen des ind. praet. der schwachen verba mit solchen des ind. praes. zusammengefallen; $d u$ spieltest wurde gleich du spielst, er spielle $=e r$ spielt, $i h r$ spieltel $=i h r$ spielt. Fur diese personen, insbesondere für die in der erzählung uberaus băufig gebrauchte 3. sing. praet., fehlte also eine form, welche die vergangenheit unzweidentig bezeichnete. Man suchte dafur einen ersatz und fand ihn in dem periphrastischen perf., das in seiner bedeutung dem praet. unter allen verbalformen am nächsten stand und sogar in manchen fällen mit diesem vertauscht werden konnte. So erlangte das perf. die bedeutung des praet., d. b. es wurde bei der erzählung einer vergangenen handlung gebraucht. Dies war zunächst der fall nur bei den schwachen verba und auch bei diesen nur fur drei personen. Wenn aber das perf. einmal diese bedeutung hatte, so konnte es sie naturgemäss nicht auf eine bestimmte anzahl von verben und personen beschrānken, sondern dehnte sie auf alle verba und alle personen aus. Da daneben in sehr vielen fällen auch das praet. gebraucht werden konnte, so entstanden doppelformen. Nach dem gesetze, dass die sprache unablässig be- 
müht ist, unniitze ungleichmässigkeiten zu beseitigen (Paul, Principien 2 288), wurde von diesen doppelformen eine ausgeschieden, und zwar diejenige, die den weniger festen anhalt im gedächtnis hatte, d. h. nicht so häufig gebraucht wurde (Behaghel, Deutsche spr. s. 71). Dies war aber das praeteritum.

Im Anz. fda. 20, 6 verweist Tomanetz auf einen ähnlichen versuch, den Nagl in seiner Gramm. analyse des niederösterreichischen dialekts, Wien 1886 gemacht hat. Zunächst fielen bei den schwachen verba ind. und. conj. praet. formell zusammen. 'Dem bauer, der bei seiner skeptischen anlage nur das gegenwärtige für reell erachtet, was er eben greifen kann, das zuküftige und vergangene, auch wenn ers glaubt, fur minder reell appercipiert, mag der gebrauch einer conjunctivform fur den ind. praet. so gut entsprochen Laben, dass sich bald auch beim starken verb der ind. mit dem conj. uniformierte. Formen wie het und tel arbeiteten vor; in vielen starken verba hatte der conj. von vornherein keinen umlaut (dial. $i$ funt, funtad, runtad), bei vielen war dieser überhaupt eine unmöglichkeit (z. b. liess, hiess, stieg), und das endende $e$, welches den conj. praet. von dem ind. praet. der starken verba unterscheiden sollte, fiel schon sehr frtih ab. War aber einmal bei allen verben die uniformierung des ind. und conj. praet. durchgefubrt (vgl. Schmeller, Bayr. gramm. 938 f.), dann mochten sich denn doch wider zweideutigkeiten und verlegenheiten zum öfteren eingestellt haben. Und diese bewogen das landvolk, vielleicht erst seit der ersten hälfte des 18. jhs. zur bezeichnung des ind. praet. das zweifellose und entschiedene perf. zu adoptieren.' Die psychologische voraussetzung dieser erklärung durfte wol nicht zutreffend sein. Denn mag ein mensch auch noch so grosse 'skeptische anlage' haben, eine tatsache, die er gestern oder vor einer woche wahrgenommen hat, wird er ebenso bestimmt aussprechen wie eine gegenwärtige. Je weiter abwärts man ferner in den volksklassen steigt, um so weniger begegnet man potentialen aussprichen; denn letztere entstehen doch durch vorsichtiges abwägen des ausdrucks, was der naiven denkweise des landvolkes durchaus widerspricht. Daher reden ja auch die kinder wenig im potentialen conjunctiv.

Sehr wertvoll dagegen ist der hinweis Nagls auf die lautliche 'uniformierung' des ind. praet. mit dem conj. praet. bei 
den schwachen und bei einer grossen anzahl von starken verbis. Dieser zusammenfall wurde für die umgangssprache noch stōrender als der des ind. praet. mit dem praes., weil hierdurch ein wunschsatz oder ein irrealer hypothetischer hauptsatz von einem aussagesatze in nichts unterschieden werden konnte. Auch fur die schriftsprache, welche die formen des ind. praet. beibebalten hat, war ja dieser zusammenfall so störend, dass die formen mit würde und möchte für den conj. praet. eingefluhrt wurden. In der mundart dagegen hat man den conj. praet. beibehalten und den ind. durch eine andere form ersetzt.

Gegen den versuch, die verdrängung des ind. praet. rein lautlich zu erklären, hat sich Wunderlich in seinem Deutschen satzbau s. $48 \mathrm{ff}$. ausgesprochen. Sein erster einwand geht dahin, dass man der 1. sing. grade in der umgangssprache nicht so leicht eine überwältigung durch formen anderer personen zutraut, und die starken verba noch weniger für eine solche abhängigkeit von den schwachen belege gegeben haben. Nun weist aber doch der ersatz der mhd. formen ich gibe, ich nime, durch nhd. ich gebe, ich nehme auf eine ziemlich starke beeinflussung der 1. sing. durch formen anderer personen, und ebenso findet man in der entwicklung der mhd. starken verba manche übergänge in die klasse der schwachen verba. Wie oben erwähnt, musste zudem das perf., wenu es einmal eine form zur erzählung vergangener tatsachen sein konnte, diese bedeutung auf alle verba und alle personen ausdehnen.

Statistische feststellungen, die Wunderlich verlangt, sind allerdings noch nicht gemacht; doch aus urkunden und chroniken des späteren mittelalters lässt sich nachweisen, dass der wegfall des auslautenden $e$ eingetreten war, als einfache praeteritalformen noch in ziemlicher anzahl existierten. Aber der gebrauch in schriftstücken kann hierin gar nichts beweisen; denn der schriftsteller steht unter dem einfluss eines tiberlieferten schulmässigen stiles und beharrt auf.diesem, ohne viel rucksicht auf die volksmundarten zu nehmen. Daher ist es ganz unzulässig, eine in so vielen mundarten verbreitete erscheinung aus der eigenart einiger weniger schriftsteller zu erklären, zumal wenn man bedenkt, dass zu der zeit, in der sich diese spracherscheinung entwickelt hat, eine schulbildung des volks fast gar nicht vorhanden war oder doch auf einer sehr tiefen stufe stand. 
Auch mit dem praes. historicum bat die verdrängung des praet. durch das perf. nichts zu tun. Wunderlich nimmt nämlich an, dass beide, historisches praes. und perf., ihr entstehen dem bestreben verdanken, ereignisse der vergangenheit in beziebung zur gegenwart zu setzen. Beim praes. hist. haben wir jedoch nach Paul (Princ. ${ }^{2}$ 230) eine wirkliche verrückung des standpunktes in der phantasie anzunehmen, indem man bei lebhafter erzählung den standpunkt, von dem aus man erzäblt, in die vergangenheit verlegt und daher die vergangenen ereignisse als gegenwärtig sieht und schildert. Wir haben also eine versetzung des gegenwärtig redenden in die vergangenheit und nicht umgekehrt eine versetzung der vergangenen handlung in die gegenwart. In den md. und oberd. mundarten ist das praes. hist. recht häufig bei lebhafter erzählung, während für die gewöhnliche erzählung das perf. gebraucht wird - also derselbe unterschied wie in den abrigen sprachen zwischen bistorischem praes. und praet. Das nämliche findet sich im nd. z. b. bei Fritz Reuter, wo das einfache praet. erhalten ist, bei der erzählung praes. und praet. neben einander gebraucht werden, während mit dem nicht praeteritalen perf. sehr sparsam gewirtschaftet wird (Wunderlich s. 50).

Zum schlusse weist Wunderlich noch auf die romanischen sprachen hin, in denen ebenfalls das periphrastische perf. neigung zeige, seine grenzen zu erweitern, während doch die formellen verhältnisse ganz anders gelagert seien. Doch handelt es sich hier ja nicht um den vollständigen verlust irgend welcher form, und zudem findet sich aucb in den romanischen sprachen mancher lautliche zusammenfall von formen verschiedener bedeutung.

Aus den ausfubrungen Wunderlichs folgt nur, dass sich praet. und perf. in ihrer bedeutung nahe gestanden haben, und dass deshalb bie und da eine vertauschung eintreten konnte, und dies ist auch die voraussetzung meiner erklärung. Dass aber in weiten sprachgebieten die eine form völlig verloren gegangen ist, kann hierdurch allein nicht befriedigend erklärt werden. Dies ist nur möglich, wenn in erster linie die lautliche entwieklung berticksichtigt wird.

DIEBURG (Hessen), 23. april 1894.

HANS REIS. 